\title{
CLIQUE-CONNECTING FOREST AND STABLE SET POLYTOPES
}

\author{
DENIS CORNAZ ${ }^{1}$
}

\begin{abstract}
Let $G=(V, E)$ be a simple undirected graph. A forest $F \subseteq E$ of $G$ is said to be clique-connecting if each tree of $F$ spans a clique of $G$. This paper adresses the clique-connecting forest polytope. First we give a formulation and a polynomial time separation algorithm. Then we show that the nontrivial nondegenerate facets of the stable set polytope are facets of the clique-connecting polytope. Finally we introduce a family of rank inequalities which are facets, and which generalize the clique inequalities.
\end{abstract}

Keywords. Graph, polytope, separation, facet.

Mathematics Subject Classification. 05C15, 90C09.

\section{INTRODUCTION}

Let $G=(V, E)$ be a simple undirected graph (without loop or multiple edge). A stable set of $G$ is a subset of pairwise nonadjacent vertices, the stable set polytope of $G$ is the convex-hull of the characteristics vectors (in $\{0,1\}^{V}$ ) of the stable sets of $G$. This polytope has been extensively studied in litterature (see e.g. [11]).

A subset of edges $F \subseteq E$ is called a forest of $G$ if the number $k$ of the connected components of the partial subgraph $(V, F)$ of $G$ satisfies $|F|+k=|V|$. (Note that some components of $(V, F)$ may be isolated vertices.) In other words, $F$ is a forest of $G$ if and only if $|F(U)| \leq|U|-1$ for every nonempty subset of vertices $U \subseteq V$ (where $F(U)$ denotes the subset of the edges of $F$ with both vertices in $U$ ). Given a weight vector $c \in \mathbb{Z}^{E}$ associated to the edges of $G$, several well-known greedy

Received April 2, 2009. Accepted October 21, 2009.

1 LIMOS, Complexe scientifique des Cézeaux, 63177 Aubiere Cedex, France;

cornaz@isima.fr 
algorithms (e.g. Kruskal, Prim, see [11]) find a forest $F$ maximizing its weight $c(F)=\sum_{e \in F} c_{e}$ in polynomial time. The forest polytope of $G$ is the convex-hull of the characteristic vectors (in $\{0,1\}^{E}$ ) of the forests of $G$. Edmonds [5] showed that for any graph $G$, its forest polytope is described by the following system of linear inequalities:

$$
\begin{gathered}
0 \leq x_{e} \leq 1 \text { for } e \in E, \\
x(E(U)) \leq|U|-1 \text { for nonempty } U \subseteq V .
\end{gathered}
$$

Optimizing over (1)-(2) is, given a vector $c \in \mathbb{Q}^{E}$, to determine $\max c x$ over $x \in \mathbb{Q}^{E}$ satisfying (1)-(2). Separating over (1)-(2) is, given a vector $\bar{x} \in \mathbb{Q}^{E}$, to decide if $\bar{x}$ satisfies (1)-(2) or to find a constraint violated by $\bar{x}$. The greedy algorithms together with the result by Edmonds show that we can optimize over (1)-(2) in polynomial time (see e.g. [11]). A consequence of the optimization-separation theorem by [6] is that separating over (1)-(2) can be done in polynomial time. Independently, Picard and Queyranne [9] and Padberg and Wolsey [8] gave a polynomial combinatorial algorithm separating (1)-(2).

A matching of $G$ is a subset of pairwise disjoint edges. After giving a combinatorial algorithm finding a matching optimizing any linear function, Edmonds [4] showed that the matching polytope is described by the following system of linear inequalities:

$$
\begin{aligned}
x_{e} \geq 0 & \text { for } e \in E, \\
x(\delta(v)) \leq 1 & \text { for } v \in V, \\
2 x(E(U)) \leq|U|-1 & \text { for odd cardinality } U \subseteq V .
\end{aligned}
$$

(Where, as usual, $\delta(v)$ is the set of the edges of $G$ incident with the vertex $v$.)

A clique of $G$ is a subset of vertices any two of which are adjacent. Determining the minimum number of cliques in a partition of $V$ into cliques of $G$ is NP-hard, see e.g. [11] (it is equivalent to the graph coloring problem). A forest $F \subseteq E$ of $G=(V, E)$ is said to be clique-connecting if each tree of $F$ spans a clique of $G$, that is, if each connected component of the partial subgraph $(V, F)$ of $G$ induces a complete subgraph of $G$. Clique-connecting stars are considered in [1] for the representative formulation of graph coloring. It is also used, with additional restrictions, in [2] to show a one-to-one correspondence beetween the colorings of $G$ and the stable sets of $\tilde{G}$, where $\tilde{G}$ is a partial subgraph of the line graph of the complementary $\bar{G}$ of $G$.

This paper adresses the clique-connecting forest polytope of $G$, that is the convex-hull of the incidence vectors of the clique-connecting forests of $G$. A first motivation for studying that polytope is that it is a "coloring polytope", in the sense that optimizing $1^{T} x$ over it is equivalent to determining the chromatic number [2]. A second motivation for studying the clique-connecting forest polytope is that it lays beetween two well described polytopes. Indeed, it is obvious that the forest polytope of $G$ contains the clique-connecting forest polytope 
of $G$. Futhermore, since any matching of $G$ is a clique-connecting forest of $G$, the clique-connecting forest polytope contains the matching polytope.

In this paper, we give a formulation for the clique-connecting forest polytope. That is, we define a polyhedron the integer vectors of which are precisely the characteristic vectors of the clique-connecting forests. The number of linear inequalities defining the polyhedron may be exponential with respect to the size of $G$, but we give a polynomial time combinatorial algorithm separating over them. Consequently, by [6], there is a polynomial time algorithm optimizing over the polyhedron.

Then we study the facial structure of the polytope. We show that every nontrivial nondegenerate facet of the stable set polytope corresponds to a facet of the clique-connecting forest polytope. Futhermore, we give a set of rank facets of the clique-connecting forest polytope which cannot be deduced from facets of the stable set polytope. These facets, called the $K$-complete set inequalities, are associated with each (not necessarily maximal) clique $K$ and they generalize the clique facets.

\section{Formulation And Separation}

Let $G=(V, E)$ be a graph. For each edge subset $F \subseteq E$ the characteristic vector of $F$ is the vector $x \in\{0,1\}^{E}$ such that for each $e \in E$, then $x_{e}=1$ if $e \in F$, and $x_{e}=0$ if $e \notin F$. We claim that an integer vector $x \in \mathbb{Z}^{E}$ is the characteristic vector of a clique-connecting forest of $G$ if and only if $x$ satisfies:

$$
\begin{gathered}
\qquad \leq x_{e} \leq 1 \text { for each } e \in E, \\
x(E(U)) \leq|U|-\left\{\begin{array}{l}
1 \text { if } U \text { is a clique of } G \\
2 \text { otherwise }
\end{array} \text { for nonempty } U \subseteq V .\right.
\end{gathered}
$$

To see sufficiency, first observe that if $x$ satisfies (6)-(7) then it satisfies (1)-(2). Hence, since $x$ is integer, it is the characteristic vector of a forest $F$ of $G$. Now if $U$ is the vertex set of a connected component of $F$, then $x(E(U))=|U|-1$. Since $x(E(U)) \leq|U|-2$ for each subset $U \subseteq V$ which is not a clique of $G$, hence $U$ is a clique of $G$. It follows that $F$ is clique-connecting. To see necessity, let $x$ be the characteristic vector of a clique-connecting forest $F$ of $G$. Clearly, $x$ is integer and satisfies (1)-(2). Given a subset $U \subseteq V$ which is not a clique of $G$, then no tree of $F$ spans $U$. It follows that $|F(U)| \leq|U|-2$, and hence $x$ satisfies (6)-(7).

Now let us consider the polyhedron defined by the vectors of $\mathbb{R}^{E}$ satisfying (6)(7). We claim that one can optimize over that polyhedron in a polynomial time. By [6] we only need to solve in polynomial time the separation problem which can be stated as follows:

Separation problem. Given $\bar{x} \in \mathbb{Q}_{+}^{E}$, decide if $\bar{x}$ satisfies (6)-(7), and if not, find an inequality violated by $\bar{x}$.

One can adapt the proof in $[8,9]$ for the separation problem associated with (1)(2) in order to prove that separating over (6)-(7) can be done in a polynomial time. 
The proof of $[8,9]$ is based on Theorem 2.1 below. For the sake of completeness we give a proof (similar to that by Schrijver in [11], Vol. B, p. 880). For any graph $G=(V, E)$ and $U \subseteq V$, let $\delta_{G}(U)$ be the cut composed by the edges of $G$ incident with one vertex in $U$ and the other vertex in $V \backslash U$ outside.

Theorem 2.1 (Rhys [10]). Given a graph $G=(V, E)$, two vectors $x \in \mathbb{Q}_{+}^{E}$ and $y \in \mathbb{Q}^{V}$, and a subset $S \subseteq V$, we can find in a strongly polynomial time a set $U$ with $S \subseteq U \subseteq V$ minimizing $x\left(\delta_{G}(U)\right)+y(U)$.

Proof. We transform the graph $G$ into a graph $H$ by the following operations: First we extend the graph by two new vertices $s$ and $t$, by new edges $\{s, v\}$ for each $v \in V$ with $y_{v}>0$, and by new edges $\{v, t\}$ for each $v \in V$ with $y_{v}<0$, so we obtain a new graph with edge set $\tilde{E}$; Then we replace the vertices in $S \cup\{s\}$ by the vertex $s$, that is, we contract $S \cup\{s\}$ (we can assume that the loops are deleted). For each $e \in E$ define the capacity $c_{e}$ of $e$ by $c_{e}:=x_{e}$. For each $\{s, v\} \in \tilde{E} \backslash E$ define the capacity $c_{e}$ of $e$ by $c_{e}:=y_{v}$. For each $\{v, t\} \in \tilde{E} \backslash E$ define the capacity $c_{e}$ of $e$ by $c_{e}:=\left|y_{v}\right|$. Then one has for any $U \subseteq V \backslash S$ :

$$
\begin{aligned}
c\left(\delta_{H}(U \cup\{t\})\right) & =x\left(\delta_{G}(U)\right)+\sum_{v \in U: y_{v}>0} y_{v}+\sum_{v \in V \backslash U: y_{v}<0}\left|y_{v}\right| \\
& =x\left(\delta_{G}(U)\right)+y(U)+\sum_{v \in V: y_{v}<0}\left|y_{v}\right| .
\end{aligned}
$$

Since $\sum_{v \in V: y_{v}<0}\left|y_{v}\right|$ is a constant, it follows that minimizing $x\left(\delta_{G}(U)\right)+y(U)$ reduces to finding a minimum-capacity cut separating $s$ and $t$ in $H$; which can be done in a strongly polynomial time with a max-flow algorithm (see e.g. [11]).

For the sake of completeness we give a proof (similar to that by Schrijver in [11], Vol. B, p. 881) of the corollary below.

Corollary $2.2[8,9]$. Given a graph $G=(V, E)$ and a vector $x \in \mathbb{Q}_{+}^{E}$, we can decide if $x$ satisfies (1)-(2) (that is, if $x$ belongs to the forest polytope of $G$ ), and if not, find the most violated inequality among (1)-(2), in strongly polynomial time.

Proof. We can assume that $x$ satisfies (1). Define $y_{v}:=2-x(\delta(\{v\}))$ for $v \in V$. Then $2(x(E(U))-|U|)=-x(\delta(U))-y(U)$. So any set $U \subseteq V$, such that $U$ contains a given vertex $u$, minimizing $x(\delta(U))+y(U)$, maximizes $x(E(U))-|U|$. By Theorem 2.1 (with $S=\{u\}$ ), we can find such a $U$ in polynomial time. Hence, by finding $|V|$ such sets, one for each $u \in V$, we can assume that $U$ is a nonempty subset of $V$ maximizing $x(E(U))-|U|$. If $x(E(U))-|U| \leq-1$, then $x$ satisfies (2), and otherwise $U$ gives a most violated inequality.

Now we can state Corollary 2.3 below, which implies that optimizing over (6)(7) is polynomial.

Corollary 2.3. The separation problem for (6)-(7) can be solved in polynomial time. 


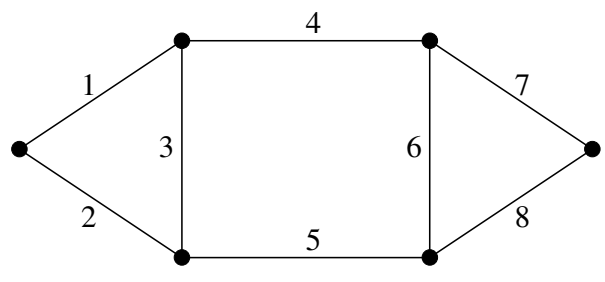

Figure 1. A graph $G=(V, E)$ with $E=\{1,2,3,4,5,6,7\}$.

Proof. By Corollary 2.2, we can assume that $x$ satisfies (1)-(2). Hence we only need to separate

$$
x(E(U)) \leq|U|-2 \text { for each subset } U \subseteq V \text { which is not a clique of } G .
$$

Let $\mathcal{S}$ be the set of the stable sets of $G$ with cardinality 2 . Note that the cardinality of $\mathcal{S}$ is polynomial. As in Corollary 2.2, we define $y_{v}:=2-x(\delta(\{v\}))$ for $v \in V$. So, for any $S \in \mathcal{S}$, any set $U$ with $S \subseteq U \subseteq V$ minimizing $x(\delta(U))+y(U)$ maximizes $x(E(U))-|U|$. By Theorem 2.1, we can find such a $U$ in polynomial time. By finding $|\mathcal{S}|$ such sets, one for each $S \in \mathcal{S}$, we can assume that $U$ is a nonempty subset of $V$ maximizing $x(E(U))-|U|$. If $x(E(U))-|U| \leq-2$, then $x$ satisfies (8), and otherwise $U$ gives a violated inequality.

\section{FACETS}

In this section we focus on the vectors of the polyhedron (6)-(7) which are not in the clique-connecting polytope of $G$. Our aim is to find new valid inequalities, that is, cutting some of these vectors out of the polyhedron but keeping the vectors of the polytope in the polyhedron.

First we remark that if each component of $G$ is a complete graph, then the clique-connecting forests of $G$ are the forests of $G$, so (6)-(7) is equal to (1)(2) and it decribes the clique-connecting forest polytope of $G$. Futhermore, we remark that if $G$ has no triangle, then the clique-connecting forests of $G$ are the matchings of $G$ and so the polytope is described by (3)-(5). Finally, we note that the clique-connecting polytope is full-dimensional, since it contains the matching polytope.

Now let us consider for instance the graph of Figure 1.

Assume that the edges 4 and 5 have a weight $c_{4}=c_{5}=3$, and that the other edges have a weight 2 . It is easily seen that the maximum of $c x$ over $x$ in the clique-connecting forest polytope is 8 (the maximum is obtained by a forest with 4 edges with weight 2). However the maximum of $c x$ over $x$ satisfying (6)-(7) is strictly greater since the vector $\bar{x}$ with $\bar{x}_{e}=1 / 2$ for each $e \in E$ satisfies (6)-(7) and it gives $c \bar{x}=9$. Now let us give a description of the clique-connecting forest polytope of the graph of Figure 1. It has 18 facets each of which is defined by one 
of the 18 linear inequalities below:

$$
\begin{aligned}
& x_{1}, \quad x_{2}, \quad x_{3}, \quad x_{4}, \quad x_{5}, \quad x_{6}, \quad x_{7}, \quad x_{8} \geq 0 \\
& \begin{array}{lll}
x_{1} & +x_{4} & \leq 1
\end{array} \\
& \begin{array}{lll}
x_{2} & +x_{5} & \leq 1
\end{array} \\
& x_{3}+x_{4} \quad \leq 1 \\
& x_{3}+x_{5} \quad \leq 1 \\
& x_{4} \quad+x_{6} \quad \leq 1 \\
& \begin{aligned}
x_{4} \quad+x_{7} & \leq 1 \\
& \leq 1
\end{aligned} \\
& \begin{array}{lr}
x_{5}+x_{6} & \leq 1 \\
x_{5} & +x_{8} \leq 1
\end{array} \\
& \begin{aligned}
x_{1}+x_{2}+x_{3}+x_{4}+x_{5} & \leq 2
\end{aligned} \\
& x_{4}+x_{5}+x_{6}+x_{7}+x_{8} \leq 2 \text {. }
\end{aligned}
$$

(To see this first note that the 18 inequalities are valid and that they imply (6)(7). So, one must check that each vector satisfying at least 8 linearly independant inequalities with equality among the 18 is integer.) Of course the vector $\bar{x}$ with components $1 / 2$ does not belong to the polytope. Indeed, it violates the last two constraints with right-hand-side 2. (The other constraints are of type (6)(7).) One can already remark that the inequalities of type $x_{e} \geq 0$, namely the nonnegativity constraints, always define facets. Indeed, these are already facets of the forest polytope and of the matching polytope. To make it even clearer, the vector $x=0$ together with the vectors $x_{f}$ with all components 0 except $\bar{x}_{f}=1$ for $f \in E \backslash\{e\}$ form $|E|$ affinely independant vectors in the clique-connecting forest polytope such that $x_{e}=0$.

In a general context, a valid inequality with $0-1$ coefficients and integer rightand-side (up to multiplying by a scalar) is called a rank inequality. For instances, (2) for the forest polytope, and (4)-(5) for the matching polytope. A rank inequality with right-and-side 1 is called a clique inequality. A clique inequality is said to be maximal if it is not dominated by another clique inequality. Very often, e.g. the stable set polytope [7], the maximal clique inequalities define facets. In contrast, a rank inequality generally does not define a facet even if it is dominated by no other rank inequality. For instance for the clique-connecting forest polytope of the graph of Figure 1: $\sum_{i=1}^{i=8} x_{i} \leq 4$. Futhermore, of course, the rank inequalities does not describe the polytope in general, e.g. the wheel inequalities are non-rank facets of the stable set polytope [3].

In the following we enlight a strong link beetween the clique-connecting forest polytope and the stable set polytope.

For any vertex $u$ of $G$, let $N(u)$ be the set of the vertices in $V$ adjacent with $v$. For any $F \subseteq \delta(u)$, we let $N_{F}(u)$ denotes the set of the vertices in $N(u)$ incident with an edge in $F$. For any $U \subseteq V$, let $G_{U}$ be the subgraph of $G$ induced by the vertices in $U$. As usual, $\bar{G}$ is the complementary graph of $G$. It is not hard to see that this lemma holds:

Lemma 3.1. For any vertex $u$ of $G, F \subseteq \delta(u)$ is a clique-connecting forest of $G$ if and only if $N_{F}(u)$ is a clique of $G_{N(u)}$, that is, a stable set of $\bar{G}_{N(u)}$. 
Note now that, given a vertex $u$, there is a natural one-to-one correspondence beetween the vectors in $\mathbb{R}^{\delta(u)}$ and the vectors in $\mathbb{R}^{N(u)}$. It follows from the above lemma that a vector $x \in \mathbb{R}^{E}$ with $x_{e}=0$ for each $e \in E \backslash \delta(u)$ is in the cliqueconnecting forest polytope of $G$ if and only if its corresponding vector in $\mathbb{R}^{N(u)}$ is in the stable set polytope of $\bar{G}_{N(u)}$. A facet of a polytope is called trivial if it has at most one nonzero coefficient. For instance the trivial facets of the stable set polytope are those of the form $x_{v} \geq 0$ or, if $v$ is isolated, $x_{v} \leq 1$. Suppose that $G$ is composed of tree vertices $u, v, w$ and two edges $u v, v w$. Then the facets of the clique-connecting forest polytope of $G$ corresponds to the stable set polytope of $\bar{G}_{N(v)}$ but not to the the facets of the stable set polytope of $\bar{G}_{N(u)}$. Indeed, $x_{v} \leq 1$ is a trivial facet of this polytope but $x_{u v} \leq 1$ is not a facet of the clique-connecting forest polytope. Suppose now that $G$ is the graph of Figure 1. One can observe that each of the eight facets with two nonzero coefficients of the clique-connecting forest polytope is one of the two nontrivial facets of the stable set polytope of $\bar{G}_{N(u)}$, where $u$ is one of the four degree 3 vertices.

Let us define one classe of facets:

Definition 3.2. Let $G=(V, E)$ be a graph and let $\sum_{u \in V} a_{u} x_{u} \leq \alpha$ be an inequality which defines a nontrivial facet of the stable set polytope of $G$. If there exists two nonadjacent vertices $v, w$ of $G$ such that every stable set $S$ with $\sum_{u \in S} a_{u}=\alpha$ contains either $v$ or $w$, then the facet is called degenerate.

Note that classical nontrivial facets, namely the clique inequalities, the oddcycle inequalities and the wheel inequalities, are nondegenerate. Actually the following problem is open:

Open problem 1. Is there a graph the stable set polytope of which has degenerate facets?

Theorem 3.4 below shows that for each vertex $v$, each nontrivial and nondegenerate facet of the stable set polytope of $\bar{G}_{N(v)}$ is a facet of the clique-connecting forest polytope. In order to prove the theorem we need the following lemma.

Lemma 3.3. Let $G=(V, E)$ be a graph and let $\sum_{v \in V} a_{v} x_{v} \leq \alpha$ be an inequality which defines a nontrivial and nondegenerate facet of the stable set polytope of $G$. Then

(i) For any vertex $v$ of $G$, there exists a stable set of $G$ whose incidence vector is in the facet, which does not contains $v$.

(ii) For any nonadjacent vertices $v$ and $w$ of $G$, there exists a stable set of $G$ which contains neither $v$ nor $w$, and the incidence vector of which is in the facet.

Proof. ( $i$ ) If every stable set whose incidence vector is in the facet contains the vertex $v$, then the facet is included in the hyperplan $x_{v}=1$. Since the stable set polytope is full-dimensional, it follows that $\sum_{v \in V} a_{v} x_{v} \leq \alpha$ is equivalent to the trivial valid inequality $x_{v} \leq 1$, leading to a contradiction.

(ii) Trivial since the facet is nondegenerate.

Now we can prove Theorem 3.4. 
Theorem 3.4. Let $u$ be a vertex of $G$, and let us denote each edge uv in $\delta(u)$ by $e_{v}$. If an inequality $\sum_{v \in N(u)} a_{v} x_{v} \leq \alpha$ defines a nontrivial and nondegenerate facet of the stable set polytope of $\bar{G}_{N(v)}$, then the corresponding inequality

$$
\sum_{e_{v} \in \delta(u)} a_{v} x_{e_{v}} \leq \alpha
$$

defines a facet of the clique-connecting forest polytope of $G$.

Proof. By Lemma 3.1, (9) is valid for the clique-connecting forest polytope. We only need to show that there are $|E|$ affinely independant vectors $x$ in the cliqueconnecting forest polytope satisfying (9) with equality. There are already $d:=$ $|\delta(u)|$ such vectors, since $\sum_{v \in N(u)} a_{v} x_{v} \leq \alpha$ defines a facet of the stable set polytope of $\bar{G}_{N(u)}$, which is full-dimensional. We can take the $d$ vectors in $\{0,1\}^{\delta(u)}$ and we let $F_{1}, \ldots, F_{d} \subseteq \delta(u)$ be the corresponding clique-connecting forests. Now let $e \in E \backslash \delta(u)$. We claim that there is some $i \in\{1, \ldots, d\}$ such that $F_{i} \cup\{e\}$ is a clique-connecting forest. If the claim holds, it makes $|E|$ affinely independant vectors satisfying (9) with equality and the proof is done. If $e$ is incident with no vertex in $N(u)$ the claim is clearly true. If $e$ is incident with one vertex $v$ in $N(u)$, by Lemma 3.3(i), we can assume that there is a $\mathrm{n} F_{i}$ which does not contains $e_{v}$. Hence $F_{i} \cup\{e\}$ is a clique-connecting forest. We can suppose now that $e$ is incident with two vertices $v$ and $w$ in $N(u)$. By Lemma 3.3(ii), there is an $F_{i}$ which contains neither $e_{v}$ nor $e_{w}$. Hence $F_{i} \cup\{e\}$ is a clique-connecting forest. Hence the claim holds, and then the theorem is true also.

Our aim for the rest of the paper is to present a family of rank inequalities defining facets of the clique-connecting forest polytope and which are not facet of the stable set polytope of $\bar{G}_{N(u)}$ for some vertex $u$. The rank of a subset of edges $E^{\prime}$ of $G$, denoted by $r\left(E^{\prime}\right)$, is equal to the maximum cardinality of a clique-connecting forest $F$ of $G$ such that $F \subseteq E^{\prime}$. So, a rank inequality for the clique connectingforest polytope of $G$ is an inequality $x\left(E^{\prime}\right) \leq r\left(E^{\prime}\right)$ for some $E^{\prime} \subseteq E$. Let us identify briefly the most natural ones. First, it is not hard to see that a subset of edges $E^{\prime}$ of $G$ has rank 1 if and only if it is an induced star of $G$ (that is, there is a stable set $\left\{v_{1}, \ldots, v_{\left|E^{\prime}\right|}\right\}$ of $G$ and a vertex $u$ such that $\left.E^{\prime}=\left\{u v_{i}: i=1, \ldots,\left|E^{\prime}\right|\right\}\right)$. Second, for every $v \in V$, the rank of $\delta(u)$ is equal to the maximum cardinality of a clique of $G_{N(u)}$. Finally, for every $U \subseteq V$, then $r(E(U))=|U|-\bar{\chi}\left(G_{U}\right)$, where $\bar{\chi}\left(G_{U}\right)$ is the minimum number of cliques in a clique partition of $G_{U}$. In the following we present a family of rank inequalities associated with edge subsets which are not necessarily of the form $\delta(u)$ for some $u \in V$ or of the form $E(U)$ for some $U \subseteq V$. The form of these subsets is described in the definition below:

Definition 3.5. Let $K \subseteq V$ be a clique of $G$ with at least two vertices and let $E(K)$ be the set of the edges of $K$. A subset $Q \subseteq E$ containing $E(K)$ is said to be a $K$-complete set of $G$ if it is an inclusionwise maximal subset such that:

(i) Every edge in $Q$ is incident with a node in $K$. 
(ii) If there exist two edges in $Q \backslash E(K)$ incident to a same vertex in $K$, then the other extremities of the two edges are nonadjacent in $G$.

(iii) For every edge in $Q$ with one vertex $v \notin K$, there exists at least one vertex $u \in K$ which is incident to no edge in $Q \backslash E(K)$ and such that $u$ and $v$ are nonadjacent in $G$.

The complete set inequalities are

$$
x(Q) \leq|K|-1 \quad \text { for every clique } K \text { with }|K|>1 \text { and every } K \text {-complete set } Q \text {. }
$$

Notice that the $K$-clique-complete sets with $|K|=2$ are precisely the maximal induced stars. So, the complete set inequalities generalize the clique inequalities.

Example 3.6. For the clique-connecting forest polytope of the graph of Figure 1, the complete set inequalities are precisely the 10 facets with non-zero right-handside.

Proposition 3.7 below states the main property of $K$-complete sets, which asserts that the complete set inequalities are valid.

Proposition 3.7. Let $K$ be a clique with at least two vertices and let $Q \subseteq E$ be a subset with $E(K) \subseteq Q$. Then $r(Q)=|K|-1$ if and only if $Q$ satisfies (i)-(iii) of Definition 3.5.

Proof. Necessity. Suppose $r(Q)=|K|-1$. Assume that there is an edge $e \in$ $Q \backslash E(K)$ disjoint from $K$. Taking a tree $T$ spanning $K$, we have a clique-connecting forest $T \cup\{e\} \subseteq Q$. So $r(Q)>|K|-1$, which is impossible; hence $Q$ satisfies $(i)$. Suppose that there are two edges $e, f \in Q \backslash E(K)$ incident to a same vertex $w$ of $K$ whose extremities are adjacent in $G$. Taking a tree $T^{\prime}$ spanning $K \backslash\{w\}$, we have a clique-connecting forest $T^{\prime} \cup\{e, f\} \subseteq Q$. Again impossible, hence $Q$ satisfies $(i i)$. Now let $e \in Q \backslash E(K)$ be incident to $v \notin K$. Let $K^{\prime}$ be the set of the vertices of $K$ which are not adjacent with $v$. If every vertex of $K^{\prime}$ is incident with an edge in $Q \backslash E(K)$, then taking $e$, taking a tree spanning $K \backslash K^{\prime}$ and taking one edge in $Q \backslash E(K)$ for each vertex in $K^{\prime}$, one finds a clique-connecting forest with $|K|$ edges. This is a contradiction, hence $Q$ satisfies $(i i i)$.

Sufficiency. Suppose that $Q$ satisfies the three properties of Definition 3.5. Let $F$ be a clique-connecting forest of $G$ such that $F \subseteq Q$ with $|F|$ maximum, so $r(Q)=|F|$. Since $K$ is a clique, then $|F| \geq|K|-1$. To show that $|F| \leq|K|-1$, we assume that $F$ has been chosen with $|F \cap E(K)|$ maximum, and we only have to prove that $F \backslash E(K)$ is empty. Suppose on the contrary that there is an edge $v w \in F \backslash E(K)$. By $(i)$ we can assume that $w \in K$ (and $v \notin K)$. By $($ iii) there is a vertex $u \in K$ which is not adjacent with $v$ and which is incident with no edge in $Q \backslash E(K)$. Since $u$ and $v$ are not adjacent, no path in $F$ links $u$ and $w$, and in particular $u w \in E(K) \backslash F$. Since $|F \cap E(K)|$ is maximal, then $(F \backslash\{v w\}) \cup\{u w\}$ is not a clique-connecting forest. It follows, since $u$ is incident with no edge in $F \backslash E(K)$, that there is an edge $w v^{\prime} \in F \backslash E(K)$ (where $v^{\prime} \notin K$ is not adjacent with $u$ ). But then $v$ and $v^{\prime}$ are adjacent; this contradicts $(i i)$. 
Corollary 3.8. Let $K$ be a clique with at least two vertices. Then $Q$ is an inclusionwise maximal subset containing $E(K)$ with rank $|K|-1$ if and only if it is a $K$-complete set.

A consequence of the corollary is that no complete set inequalities is dominated by another rank inequality. Now we can state the main result of the section.

Theorem 3.9. Let $G$ be a graph. Each complete set inequality (10) defines a facet of the clique-connecting forest polytope of $G$

Proof. Let $K$ be a clique of $G$ with at least two vertices and $Q$ a $K$-complete set. Since by Proposition 3.7, $r(Q)=|K|-1$, then inequality (10) is valid. To see that (10) determines a facet, let $\sum_{e \in E} a_{e} x_{e}=\beta$ be satisfied by all $x$ in the cliqueconnecting forest polytope of $G$ with $x(Q)=|K|-1$. We only need to prove that $\sum_{e \in E} a_{e} x_{e}=\beta$ is some multiple of $x(Q)=|K|-1$. So $a(F):=\sum_{e \in F} a_{e}=\beta$ for each clique-connecting forest $F$ with $|F \cap Q|=|K|-1$. If $|K|=2$, there is a cliqueconnecting forest $\{e\}$ where $e$ is the unique edge in $E(K)$, then $a_{e}=\beta$. Otherwise let $e_{1}, e_{2}$ be two distinct edges in $E(K)$. Since $K$ is a clique, we can assume that there exists two trees spanning $K$, say $T_{1}$ and $T_{2}$, such that $T_{1} \backslash T_{2}=e_{1}$ and $T_{2} \backslash T_{1}=e_{2}$. Then $a\left(T_{1}\right)-a\left(T_{2}\right)=a_{e_{1}}-a_{e_{2}}=\beta-\beta=0$. It follows that $a_{e}=\beta /(|K|-1)$ for every $e \in E(K)$. (If $|K|=2$ this still holds.) Now let $e \in Q \backslash E(K)$. Since every edge in $Q$ is incident to at least one node in $K$, we can suppose that $e=v w$ with $w \in K$. Let $T$ be a spanning tree of $K \backslash\{w\}$. Since $F=T \cup\{e\}$ is a clique-connecting forest such that $|F \cap Q|=|K|-1$, $a(F)=a(T)+a_{e}=\beta$. Since $a(T)=(|K|-2) \times \beta /(|K|-1)$, then $a_{e}=\beta /(|K|-1)$. Also, $a_{f}=0$ for each $f \in E \backslash Q$. Indeed, since by Corollary 3.8, $Q$ is maximal, then $r(Q \cup\{f\})=r(Q)+1$, and then there is a clique-connecting forest $F$ such that $F \backslash Q=\{f\}$ and $|F \cap Q|=|K|-1$, and hence $a(F)=\beta=a(F \backslash\{f\})$. So $a_{f}=0$. In conclusion, $\sum_{e \in E} a_{e} x_{e}=\beta$ is some multiple of $x(Q)=|K|-1$, and hence (10) define facets.

As it is implied by the proposition below, unsurprisingly, one cannot separate the complete set inequalities.

Proposition 3.10. It is NP-complete to separating the clique inequalities.

Proof. Recall that $x(Q) \leq 1$ is a clique inequality if and only if $Q$ is a maximal induced star of $G$. Hence if we could separate the clique inequalities in polynomial time, we could decide if $G$ contains an induced star with a weight $>1$ with respect to a weight function $x$. In particular we could find an induced star with maximum cardinality in polynomial time. Suppose that we want to find a stable set with maximum cardinality in a graph $H$. It suffices to finding an induced star with maximum cardinality in the graph obtained from $H$ by adding a (universal) vertex adjacent to all the other vertices of $H$. Since finding a maximum cardinality stable set is NP-hard, it follows that separating the clique inequalities is NP-hard. 


\section{Conclusion}

We have studied a coloring polytope, namely the clique-connecting forest polytope, and we have showed its link with the stable set polytope. In light of the 1-to-1 correspondence in [2], this is not too surprising to have a stronk link between these two polytopes. An open problem raises naturally concerning the clique-connecting polytope: Does this polytope have other rank facets than the ones mentionned in the present paper? Also, concerning the stable set polytope, we raised one open problem: Can it have degenerate facet? Where a "degenerate facet" is a nontrivial one with a pair of nonadjacent vertices intersecting every stable set saturating the facet.

\section{REFERENCES}

[1] M. Campêlo, R. Corrêa and Y. Frota, Cliques, holes and the vertex coloring polytope. Inf. Process. Lett. 89 (2004) 159-164.

[2] D. Cornaz and V. Jost, A one-to-one correspondence beetween stables sets and colorings. Oper. Res. Lett. 36 (2008) 673-676.

[3] E. Cheng and W.H. Cunningham, Wheel inequalitites for stable set polytopes. Math. Program. $\mathbf{7 7}$ (1997) 389-421.

[4] J. Edmonds, Maximum matching and a polyhedron with 0,1-vertices. Journal of Resarch National Bureau of Standards Section B 69 (1965) 67-72.

[5] J. Edmonds, Matroids and the greedy algorithm. Math. Program. 1 (1971) 127-136.

[6] M. Grötschel, L. Lovàsz and A. Schrijver, The ellipsoid method and its consequences in combinatorial optimization. Combinatorica 1 (1981) 169-197.

[7] M.W. Padberg, On the facial structure of set packing polyhedra. Math. Program. 5 (1973) 199-215.

[8] M.W. Padberg and L.A. Wolsey, Fractional covers for forests and matchings. Math. Program. 29 (1984) $1-14$.

[9] J.-C. Picard and M. Queyranne, Selected applications of minimum cuts in networks. INFOR Can. J. Oper. Res. Inf. Process. 20 (1982) 394-422.

[10] J.M.W. Rhys, A selection problem of shared fixed costs and network flows. Manag. Sci. 17 (1970) 200-207.

[11] A. Schrijver, Combinatorial Optimization. Springer-Verlag, Berlin, Heidelberg (2003). 